\title{
Correction to: Quark-Gluon plasma and heavy-ion phenomenology
}

Munshi G. Mustafa ${ }^{a}$

Theory Division, Saha Institute of Nuclear Physics, HBNI, 1/AF Bidhan Nagar, Kolkata 700064, India

Published online 18 June 2021

(C) EDP Sciences, Springer-Verlag GmbH Germany, part of Springer Nature 2021

\section{Correction to: Eur. Phys. J. Spec. Top.}

https://doi.org/10.1140/epjs/s11734-021-00018-y

The editorial Quark-Gluon plasma and heavy-ion phenomenology, written by Munshi G. Mustafa, was accidentally published without hyperlinks to the references.

The original article has been corrected and no other changes have been made.

The original article can be found online at https://doi.org/ 10.1140/epjs/s11734-021-00018-y.

\footnotetext{
${ }^{\mathrm{a}}$ e-mail: munshigolam.mustafa@saha.ac.in (corresponding author)
} 